\title{
Nucleation and Grain Growth in Interstellar Space
}

\author{
Bertram DonN \\ NASA Goddard Space Flight Center \\ Greenbelt, Maryland
}

THE FIRST DETAILED STUDIEs to determine the processes by which I interstellar grains may form were made by a group of Dutch astronomers in the 1940's. (See refs. 1 to 5.) Since that time very little systematic work on this problem has been done until very recently when Hoyle and Wickramasinghe (ref. 6) investigated graphite formation in cool stellar atmospheres. Van de Hulst's paper in 1949 (ref. 5) represents the culmination of an intensive attack which had considerable influence on astronomical thought about interstellar grains.

Somewhat ironically, beginning about 1949 many significant advances in physics and chemistry having a direct bearing on this problem were made. In 1949, Frank in reference 7 presented a theory which explained how real crystals tend to grow, and much work, both theoretical and experimental, has been done since then. (See ref. 8.) Recent extensive research in chemical reactions at low temperatures both in solids and on surfaces is reported in reference 9. These investigations prompted renewed interest in investigating the origin of interstellar grains, and the present paper discusses part of the continuing study. Developments in stellar and galactic evolution indicate that a continuous source of grains is necessary. The interchange of interstellar material that is needed to explain the rate of star formation was studied in references 10 and 11 , and a turnover time of $10^{9}$ years was estimated. The fact that this time is short compared with the age of the galaxy, which is of the order of $10^{10}$ years, indicates that the interstellar material has been pretty well circulated through stars. Primordial grains that formed at some early stage of the galaxy should have disappeared. Other destructive processes such as collisions and passage near hot stars further shorten the lifetime.

This paper deals with the question of nucleation and subsequent growth in interstellar space. When ter Haar considered this problem in 1944, the accepted kinetic temperature for the interstellar gas was 
$10000^{\circ} \mathrm{K}$. Since the "temperature" corresponding to the internal energy of molecules and grains would be less $100^{\circ} \mathrm{K}$, he was faced with a situation representing an extreme deviation from thermodynamic equilibrium. It was necessary, therefore, to resort to a mechanistic treatment of particle formation with all the complications and uncertainties of such a method.

Today investigators face a different problem because of the recognition (ref. 12) that extensive low temperature regions occur, with kinetic temperatures near $100^{\circ} \mathrm{K}$. It is in these $\mathrm{H}$ I regions where all temperatures are below $100^{\circ} \mathrm{K}$ that grain formation takes place. Although thermodynamic equilibrium does not hold exactly, the temperatures for all degrees of freedom are sufficiently close that thermodynamic results may be expected to yield reasonable answers.

\section{NUCLEATION THEORY}

Comprehensive discussions and nucleation theory are given in references 13 to 15 , and a more concise treatment including later developments as well as crystal growth is discussed in reference 8 .

The rate of nucleation in a vapor is determined by the rate at which single molecules add to clusters of critical size to form stable nuclei. The frequency distribution of clusters and the condition of stability is determined by the free energy of formation of a cluster because entropy as well as energy changes must be accounted for. For the frequency distribution, one finds

$$
m_{i}=m \exp \left(-\Delta G_{i} / k T\right)
$$

where

$m_{i} \quad$ number of clusters of $i$ molecules per $\mathbf{c m}^{3}$

$m \quad$ number of molecules per $\mathbf{c m}^{3}$

$\Delta G_{i} \quad$ free energy of formation of $i$ th cluster

$k \quad$ Boltzmann constant

$T$ temperature

The factor $\Delta G_{i}$ may be represented by the equation

where

$$
\Delta G_{i}=4 \pi r^{2} \sigma+4 / 3 \pi r^{3} \Delta G_{v}
$$

$\sigma \quad$ surface energy

$r \quad$ nuclear radius (assumed spherical)

$\Delta G_{v}$ free energy difference per unit volume of liquid between supersaturated vapor at ambient pressure $P$ and bulk liquid at its equilibrium vapor pressure

The term $\Delta G_{v}$ is given by

$$
\Delta G_{v}=-\frac{k T}{\Omega} \ln \frac{P}{P_{v}}
$$


where

$\Omega$ molecular volume

$P \quad$ ambient pressure

$P_{v} \quad$ equilibrium vapor pressure of bulk phase

One of the weak points of the theory is the assumption that macroscopic thermodynamic properties apply to the small clusters. Equations (1) and (3) hold for crystals as well as drops when the necessary changes in defining the various quantities have been made.

Initially, $\Delta G_{i}$ increases as molecules add to a cluster, with the result that clusters represent unstable fluctuations that form and decay. Equation (3) shows that the second term in equation (2) is negative, and the free energy of a cluster has a maximum value obtained by setting

$$
\frac{\mathrm{d} \Delta G_{i}}{\mathrm{~d} r}=0
$$

This procedure yields the characteristics of the cluster of critical size for which further growth decreases the free energy. Clusters larger than the critical size will not tend to decay spontantously and are the condensation nuclei.

The critical parameters are as follows:

$$
\begin{aligned}
r^{*} & =\frac{2 \sigma \Omega}{k T \ln \left(P / P_{v}\right)} \\
i & =\frac{4 \pi r^{3}}{3 \Omega} \\
\Delta G^{*} & =\frac{16 \pi \sigma^{3}}{3(k T / \Omega)^{2} \ln ^{2}\left(P / P_{v}\right)}
\end{aligned}
$$

The nucleation rate is the rate at which molecules collide with and stick to clusters of the critical size. This rate is given by:

where

$$
J=\alpha Z 4 \pi\left(r^{*}\right)^{2} \frac{P}{(2 \pi m k T)^{1 / 2}} m \exp \left(-\Delta G^{*} / k T\right)
$$

$J$ number of nuclei formed per $\mathrm{cm}^{2}$ per sec

$\alpha$ sticking coefficient

$Z$ nonequilibrium factor

The factor $Z$ arises because critical clusters are reduced from their equilibrium concentration by the growth of stable nuclei into droplets or crystallites. 


\section{INTERSTELLAR NUCLEATION}

Equations (3) to (7) show the fundamental role of the supersaturation ratio $P / P_{v}$ in the nucleation process. Consequently, this ratio must be determined in the interstellar medium for all elements of interest. The partial pressure $P_{i}$ of the $i$ th element is given by

$$
P_{i}=m_{i} k T
$$

where $m_{i}$ is the interstellar concentration of the element. Table I displays concentrations, partial pressures at $100^{\circ} \mathrm{K}$, and impact frequencies per $\mathrm{cm}^{2}$ for the more abundant elements and observed radicals. Relative abundances are from reference 16; uncertainties of these values are not sufficient to affect the results of this analysis.

\section{TABLE I.-The Interstellar Gas}

\begin{tabular}{|c|c|c|c|c|}
\hline Element & $\begin{array}{c}\text { Atomic } \\
\text { number }\end{array}$ & $\begin{array}{c}m_{i}, \\
\text { atoms } / \mathrm{cm}^{3}\end{array}$ & $\begin{array}{c}P_{i}, \\
\text { torr }\end{array}$ & $\begin{array}{c}\text { Impact } \\
\text { frequency } \\
\text { no. } / \mathrm{cm}^{2} \cdot \mathbf{s e c}\end{array}$ \\
\hline $\mathrm{H}$ & 1 & 1 & $10^{-17}$ & $4 \times 10^{4}$ \\
$\mathrm{He}$ & 4 & $1 \times 10^{-1}$ & $10^{-18}$ & $2 \times 10^{3}$ \\
$\mathrm{O}$ & 8 & $5 \times 10^{-4}$ & $5 \times 10^{-21}$ & 5 \\
$\mathrm{Ne}$ & 10 & $2 \times 10^{-4}$ & $2 \times 10^{-21}$ & 2 \\
$\mathrm{~N}$ & 7 & $2 \times 10^{-4}$ & $2 \times 10^{-21}$ & 2 \\
$\mathrm{C}$ & 6 & $10^{-4}$ & $10^{-21}$ & 1 \\
$\mathrm{Si}$ & 14 & $3 \times 10^{-5}$ & $3 \times 10^{-22}$ & $2 \times 10^{-1}$ \\
$\mathrm{Mg}$ & 12 & $2 \times 10^{-5}$ & $2 \times 10^{-22}$ & $2 \times 10^{-1}$ \\
$\mathrm{Fe}$ & 26 & $2 \times 10^{-5}$ & $2 \times 10^{-22}$ & $10^{-1}$ \\
$\mathrm{~S}$ & 16 & $10^{-4}$ & $10^{-22}$ & $7 \times 10^{-2}$ \\
$\mathrm{Ar}$ & 18 & $3 \times 10^{-6}$ & $3 \times 10^{-23}$ & $3 \times 10^{-2}$ \\
$\mathrm{Al}$ & 13 & $3 \times 10^{-6}$ & $3 \times 10^{-23}$ & $2 \times 10^{-2}$ \\
$\mathrm{Ca}$ & 20 & $10^{-6}$ & $10^{-23}$ & $6 \times 10^{-3}$ \\
$\mathrm{Na}$ & 11 & $10^{-6}$ & $10^{-23}$ & $7 \times 10^{-3}$ \\
$\mathrm{Ni}$ & 28 & $10^{-6}$ & $10^{-23}$ & $5 \times 10^{-3}$ \\
$\mathrm{CH}, \mathrm{CN}$, & & $10^{-8}$ & $10^{-25}$ & $10^{-4}$ \\
$\mathrm{OH}$ & & $10^{-7}$ & $10^{-24}$ & $10^{-3}$ \\
\hline
\end{tabular}

The elements to which this calculation applies and whose vapor pressure is of interest are carbon, silicon, and the metals which condense directly and require no recombination reactions first. These are all of low volatility, and vapor pressures cannot be measured at low temperatures.

Vapor pressures can be calculated theoretically by using capacities for the solid and molecular energy levels for the vapor. The procedure is discussed in references 17 to 19 . The resulting equation for the vapor pressure is 


$$
\ln P_{v}=-\frac{L_{0}}{R T}+\frac{5}{2} \ln T-\int_{0}^{T} \frac{\mathrm{d} T}{R T^{2}} \int_{0}^{T}\left(C_{p_{s}}-C_{i}\right) \mathrm{d} T+i
$$

where

$L_{0}$ heat of vaporization at $0^{\circ} \mathrm{K}$

$C_{p_{s}}$ specific heat of solid

$C_{i}$ internal specific heat of gas

$h$ Planck's constant

$i$ chemical constant $=\ln g_{0} \frac{(2 \pi m)^{3 / 2} k^{5 / 3}}{h^{3}}$

$m$ molecular mass

$g_{0}$ statistical weight of molecule

$R$ gas constant

Vapor pressures, listed as equilibrium constants for solid-vapor transition, are given in reference 20 for $100^{\circ}$ intervals starting at $100^{\circ} \mathrm{K}$. The $100^{\circ} \mathrm{K}$ data are appropriate for an $\mathrm{H} \mathrm{I}$ region. Vapor pressures $P$ and the supersaturation ratio $\rho=P / P_{v}$ in the interstellar medium are given in table II.

TABLE II. - Interstellar Pressure and Supersaturation

$$
\left[T=100^{\circ} \mathrm{K}\right]
$$

\begin{tabular}{|c|c|c|}
\hline Element & $\log P$, dynes $/ \mathbf{c m}^{2}$ & $\ln \rho$ \\
\hline & & \\
Al & -157 & 315 \\
C & -358 & 780 \\
K & -35 & 32 \\
Na & -45 & 57 \\
S & -130 & 250 \\
Si & -217 & 450 \\
\hline
\end{tabular}

In order to find the critical cluster size and number of atoms from equations (4) and (5), the molecular volume $\Omega$ and surface energy $\sigma$ for the elements under consideration must be determined. The molecular volume is given by:

where

$$
\Omega=\frac{M}{N_{0} n}
$$

$M$ atomic weight

$N_{0}$ Avogadro's number, $6 \times 10^{23}$

$n$ density of bulk material, $\mathrm{g} / \mathrm{cm}^{3}$ 
Surface energies are difficult to measure with high accuracy, although results sufficient for these calculations are available for some materials in references 21 and 22. Data for carbon are discussed in references 23 and 24. Table III presents the crystal properties for nucleation calculations.

TABLE III.-Crystal Properties

\begin{tabular}{|c|c|c|c|c|}
\hline Element & $\begin{array}{l}\text { Atomic } \\
\text { weight }\end{array}$ & $\begin{array}{c}\text { Density, } \\
\text { g/cm }\end{array}$ & $\begin{array}{l}P, \\
\text { torr }\end{array}$ & $\begin{array}{c}\text { Temperature, } \\
{ }^{\circ} \mathrm{K}\end{array}$ \\
\hline Carbon & 12 & 2.2 & $1.0 \times 10^{-23}$ & 130 to 1000 \\
\hline Iron & 56 & 7.9 & 1.2 & $>1800$ \\
\hline Silicon & 28 & 2.4 & 2.0 & 1240 \\
\hline Sodium & 23 & 1.0 & 4.0 & 150 \\
\hline Zinc & 65 & 7.1 & 1.5 & 105 \\
\hline
\end{tabular}

The application of equations (4) and (5) yields $r=1.7 \times 10^{-8}$ for sodium and $r=4 \times 10^{-9}$ for carbon with $\sigma=1000 \mathrm{ergs} / \mathrm{cm}^{2}$. In each case the value of $r^{*}$ is less than unity. A straightforward interpretation would be that a single atom acts as a nucleus for continued growth. However, the proper equivalent of such macroscopic properties of matter as surface energy, for clusters of a few molecules, is one of the stumbling blocks to the development of a rigorous nucleation theory. Although the evaluation of the critical cluster or nucleus is uncertain here, it seems reasonable to conclude that in interstellar space a nucleus will consist of a very small number of atoms. Physically, this comes about because of the low interstellar temperature which leads to a very low vapor pressure for the elements. The low temperature also causes all molecular aggregates to have high stability, and independent of an interpretation as a nucleation process we expect any diatomic molecules to be stable against spontaneous decay. As shown in reference 3 , the steady-state molecular concentration is determined by photodissociation. The photodissociation probability of the molecules will be approximately independent of size or even increase for larger molecules as longer wavelengths tend to be effective. (See ref. 25.) This behavior is quite different from the decay of clusters in nucleation where the decay probability decreases with size until a critical size corresponding to the stable nucleus is reached.

Some preliminary estimates of the formation rate of interstellar grains based on the preceding analysis can be made. This stage seems to be the same as that from which ter Haar began his analysis. However, several features show that this is not actually true. By restricting the 
analysis to crystal growth without recombination among different elements, only the heavier elements already indicated are involved. Also, the aggregates in the present study are stable molecules undergoing photodissociation, not the spontaneously disintegrating clusters. In ter Haar's paper (ref. 2), crystalline properties of the clusters play important roles. In addition to the uncertainties already noted about using these macroscopic properties for small clusters, it does not appear that the growth mechanism presented in reference 2 is consistent in assuming that it would lead to crystalline grains. This point will be considered later.

\section{INTERSTELLAR MOLECULAR PROCESSES}

In order to examine the kinetics of the formation of pure grains, carbon is used as a significant illustration, and chemical reactions involving other species are neglected. If recombination reactions incorporate other atoms the process is the alternative mechanism of ter Haar. Should the reactions remove carbon, somewhat akin to the molecular formation process proposed in reference 26 , the growth rate becomes even slower than found here and interstellar formation of crystalline grains still less likely.

In reference 27 is obtained a rate coefficient for the reaction

$$
\mathrm{C}^{+}+\mathrm{H} \rightarrow \mathrm{CH}^{+}+h \nu
$$

given by

$$
\frac{\mathrm{d} m_{\mathrm{CH}+}}{\mathrm{d} t}=10^{-18} m_{\mathrm{C}}+m_{\mathrm{H}}
$$

The inclusion of other formation and decay processes yields a steady. state value of $10^{-10}$ to $10^{-11}$ molecules $/ \mathrm{cm}^{3}$. For $\mathrm{CN}^{+}$, the estimates of a total production in $10^{9}$ years in references 3 and 27 are $5 \times 10^{-6} / \mathrm{cm}^{3}$ and $5 \times 10^{-7} / \mathrm{cm}^{3}$, respectively. These numbers neglect photodissociation, and the actual density by this process must be less. If photodissociation for $\mathrm{CN}^{+}$is comparable to that for $\mathrm{CH}^{+}$, a steady-state density of about $10^{-14} / \mathrm{cm}^{3}$ is obtained. In the absence of more direct evidence it is assumed that $\mathrm{C}_{2}$ would behave similarly.

A third atom still has a low probability of adding to a diatomic molecule as stabilization against rapid dissociation remains low. (See ref. 28.) Consequently, for the reaction

$$
\begin{gathered}
\mathrm{C}_{2}+\mathrm{C} \rightarrow \mathrm{C}_{3}+h \nu \\
\frac{\mathrm{d} m_{\mathrm{C}_{3}}}{\mathrm{~d} t}=10^{-16} m_{\mathrm{C}} m_{\mathrm{C}_{3}}
\end{gathered}
$$


When four molecules are involved the frequency with which the newly formed bond attains the recombination energy and can break becomes comparable to the reciprocal radiative lifetime. The probability of capture of a fourth carbon is somewhat less than for normal reaction probabilities and will be about normal for additional carbon atoms.

Consider the rate coefficient for the formation of four carbons

$$
\frac{\mathrm{d} m_{\mathrm{C}_{4}}}{\mathrm{~d} t}=10^{-13} m_{\mathrm{C}_{3}} m_{\mathrm{C}}
$$

For all succeeding reactions let the rate coefficients be

$$
\frac{\mathrm{d} m_{\mathrm{C}_{i+1}}}{\mathrm{~d} t}=10^{-12} m_{\mathrm{C}_{j}} m_{\mathrm{C}} j \geqslant 4
$$

These rate coefficients are given by the products $0.01 \sigma v$ (eq. (15a)) and $0.1 \sigma v$ (eq. (15b)), where $\sigma$ is the collision cross section $\left(10^{-15} \mathrm{~cm}^{2}\right)$ and $v$ is the average relative velocity $\left(10^{4} \mathrm{~km} / \mathrm{sec}\right)$.

The formation rate of a species containing $j$ atoms is given by:

$$
\frac{\mathrm{d} m_{j}}{\mathrm{~d} t}=\gamma_{j-1} m_{j-1} m_{1}-\beta_{j} m_{j}-\gamma_{j} m_{j}+\beta_{j+1} m_{j+1}
$$

The ' $\gamma$ 's are the formation rate coefficients and the $\beta$ 's are the photodissociation rates whose values have been discussed previously. An approximate solution may be obtained for the steady state by solving each equation sequence starting with the first and dropping the last term in each equation. It will turn out that the $m_{j}$ terms decrease rapidly enough to make this legitimate.

The procedure leads to the following solution for the $m_{j}$ terms:

$$
\begin{aligned}
& m_{1}=10^{-13} \\
& m_{2}=10^{-12} \\
& m_{3}=10^{-20} \\
& m_{4}=10^{-24} \\
& m_{5}=10^{-28}
\end{aligned}
$$

For successively larger molecules the frequency is multiplied by $10^{-4}$ for each additional carbon atom added until the values of the adopted coefficients change. This will probably occur in the photodissociation process and effect $\beta_{j}$, which determines the rate of destruction. Although the building process may well be more efficient than the result obtained here indicates, two neglected factors make it less efficient. 
The dissociation will not always consist of the removal of a single atom, and the broken bond in some instances will yield larger fragments than single atoms. More significant is the fact that all elements to which our mechanism applies will be largely ionized, leading to a positively charged carbon polymer and a reduction in the resultant capture rate of additional carbon ions. It follows, therefore, that the uncertainties in the calculations will probably not yield the required grain density for conducting grains, which for an extinction of 1 magnitude kpc is about $10^{-11} / \mathrm{cm}^{3}$.

Ter Haar implied in his 1944 paper that the building process may consist of a sequence of the form

$$
\mathrm{C} \rightarrow \mathrm{CH} \rightarrow \mathrm{CH}_{2} \rightarrow \mathrm{CH}_{2} \mathrm{O} \rightarrow \mathrm{CH}_{3} \mathrm{O} \rightarrow \mathrm{CH}_{3} \mathrm{NO}
$$

and so on. This is the growth mechanism suggested in references 29 and 30 which leads to the so-called Platt particle.

\section{STELLAR SOURCES}

Because of the problem of forming grains in interstellar space, other sources require consideration. A significant contribution was made by the investigation reported in reference 6 concerning graphite formation in cool carbon stars. The graphite formation problem must consider the complete chemical equilibrium in a stellar atmosphere taking all constituents into account; hydrogen is clearly the most important. Fortunately, the chemical equilibrium of the $\mathrm{CH}$ system including graphite stability was investigated in reference 31 . Seventy hydrocarbons are included, and the temperature at which graphite becomes stable as a function of the $\mathrm{C} / \mathrm{H}$ ratio for several pressures is determined. These results are shown in figure 1 , modified from the original diagram for

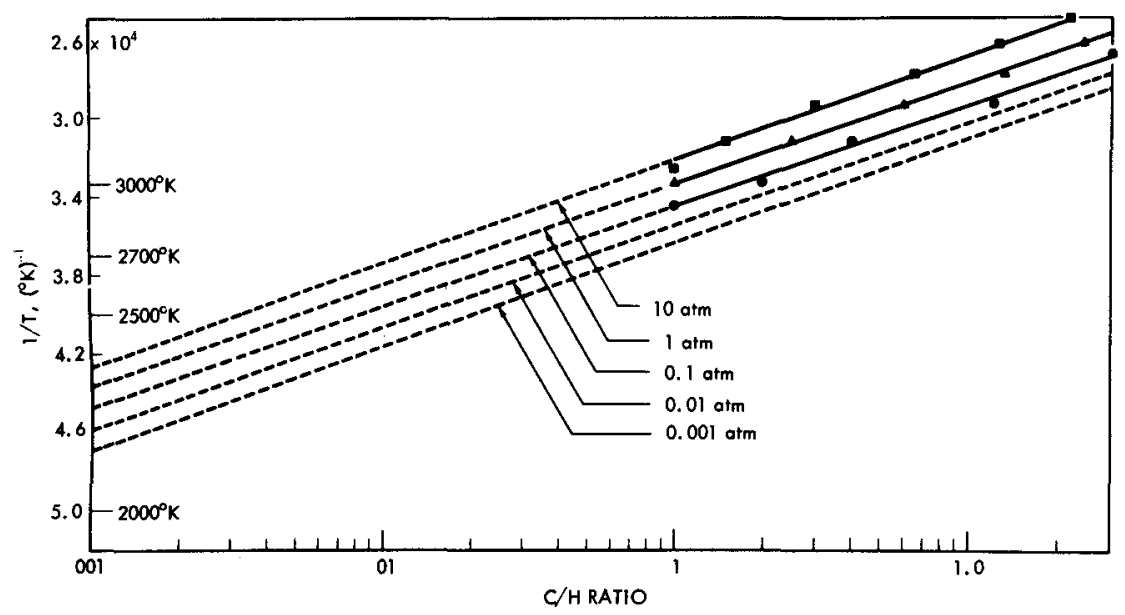

FIGURE 1.-Sublimation temperature of graphite as function of $\mathrm{C} / \mathrm{H}$ ratio of atmosphere. Points are taken from reference 31. Dashed lines are extrapolations: 
better extrapolation. Solid lines are drawn through the computed points and the dashed lines are extrapolated to a $\mathrm{C} / \mathrm{H}$ ratio of $10^{-3}$, adapted by Hoyle and Wickramasinghe. For a pressure of $10^{-3}$ atmospheres, the considerable extrapolation yields a sublimation temperature of graphite of about $2100^{\circ} \mathrm{K}$. Professor Bauer has said that the pressure extrapolation should be valid. ${ }^{1}$ At a temperature of $2100^{\circ} \mathrm{K}$, conditions are less favorable for graphite formation and the mechanism becomes rather doubtful. ${ }^{2}$

An important feature of the molecular equilibrium in carbon stars of type $\mathrm{N}$ is the array of hydrocarbon molecules and radicals present in the atmosphere. If the observed mass ejection for $M$ stars exists for carbon stars also, then a significant quantity of these species is carried into interstellar space. It is conceivable that they may serve as nuclei for further growth and that the growth process may compete favorably with photodecomposition of the molecules. As already discussed, the probability that a molecule of five or more atoms will add another is high. Whether this scheme would lead to graphitic grains or more nearly resemble the composition and structure of a Platt particle is not now clear. In examining this problem, the possibility that aggregates might develop beyond the normal atmospheric boundary, perhaps in an expanding envelope, needs to be considered.

It cannot be said that the nucleation problem has been solved. There are many uncertainties in the present discussion and several possibilities have yet to be investigated.

\section{CRYSTAL GROWTH}

With regard to particle growth on a preexisting nucleus, on the hexagonal or " $C$ " face of a graphite lattice carbon atom, bonds are highly saturated and the captured surface atom is held by the relatively weak van der Waals' forces. These nonlattice atoms would be capable of diffusing over the surface. Experiments on the formation of carbon whiskers (ref. 33) indicate that this does occur. A study of the kinetics of graphite formation in interstellar space at low temperatures (ref. 34) indicates that the captured atoms become trapped at the edge of a " $\mathrm{C}$ " face so that a platelet or needle would tend to grow. The growth rate depends upon the surface area of the grain over which atoms are collected. This produces exponential growth until the size becomes larger than the mean diffusion distance, and then linear growth occurs.

\footnotetext{
${ }^{1}$ Later work in reference 32 extended the calculations to $\mathrm{C} / \mathrm{H}=0.008$ and experimentally studied graphite equilibrium in a hydrocarbon atmosphere. The theoretical and experimental results are in agreement with the earlier work and our extrapolation. There is a suggestion that sublimation temperatures would be greater by somewhat less than $50^{\circ}$.

'See, however, the Donn comment following Wickramasinghe's paper in the present compilation concerning a recent detailed analysis of this problem by Donn, Wickramasinghe, Hudson, and Stecher.
} 
Growth of a graphite platelet on a $\mathbf{1 0}^{-7}$-cm nucleus is shown in table IV.

TABLE IV.-Growth of Graphite Platelet

\begin{tabular}{|c|c|c|}
\hline \multirow{2}{*}{ Time, yr } & \multicolumn{2}{|c|}{ Radius, for hydrogen number density of- } \\
\cline { 2 - 3 } & $1 / \mathrm{cm}^{3}$ & $10 / \mathrm{cm}^{3}$ \\
\hline $10^{7}$ & $10^{-7}$ & $10^{-6}$ \\
$10^{8}$ & $10^{-6}$ & $10^{4}$ \\
$10^{9}$ & $10^{-4}$ & $\cdots \cdots$ \\
\hline
\end{tabular}

With a density of 1 hydrogen atom $/ \mathrm{cm}^{3}$, practically no change occurs in $10^{7}$ years. In $10^{8}$ years the grain reaches a radius of $10^{-6} \mathrm{~cm}$ and the rate increases very rapidly for greater time intervals because of the exponential behavior. The figure of $10^{-4}$ in column 2 is obtained by using the exponential growth expression and obviously is meaningless except to show how rapidly the size increases once growth begins. Column 3 shows the size attained for the intervals indicated in denser regions where the hydrogen number density is $10 / \mathrm{cm}^{3}$.

It can be seen that the change in size is much greater than the density increase for grains of $10^{-6} \mathrm{~cm}$. This theory can provide for grain size distribution which changes discontinuously from small to large grains in different regions of the Galaxy. These results neglect effects of adsorbed atoms or attack by atomic hydrogen and oxygen which may remove carbon atoms.

A point related to the question of coating grains, or graphite cores with ice mantles, should be emphasized. To have an efficient substrate for condensing a different molecule, the two species should have similar crystal properties, in particular with regard to crystal structure and lattice spacing. Silver iodide has a hexagonal structure similar to ice I (normal ice) and is an effective nucleating agent for ice. A solution of silver iodide was cooled under sufficient pressure such that ice III was the stable phase in reference 35; however, ice I was again obtained. Silver iodide and ice III have dissimilar structure and spacing and silver iodide will not nucleate ice III even in the pressure-temperature domain where ice III is the stable phase. In another type of experiment (ref. 36), the sticking coefficient dropped from unity to about 0.65 as the lattice spacing mismatch increased from 0 to 15 percent for cubic crystals.

These phenomena indicate the necessity of detailed analysis of particle formation; it is not as simple as saying that material condenses. An extensive body of theoretical and experimental data exist which must be incorporated into a theory of interstellar grain formation.

271-992 O-67-14 
Much more experimental work is needed, particularly for conditions appropriate to astrophysics.

It should be emphasized that only tentative conclusions have been presented herein. A study of interstellar grain formation can now be carried out which is on a sounder basis than was possible in the period from 1940 to 1950 . It is necessary, however, to do this in succes. sive stages. This paper discussed the nucleation process in some detail and more briefly, the crystal growth process. Some attention was also given to the role of chemical reactions. A program for continuing study of the origin and structure of interstellar grains is outlined in reference 37.

\section{REFERENCES}

1. Oort, J. H.; and van De Hulst, H. C.: Gas and Smoke in Interstellar Space. Bull. Astron. Inst. Netherlands, vol. 10, 1946, p. 187.

2. Ter Haar, D.: Astrophys. J., vol. 100, 1944, p. 288.

3. Kramers, A. H.; and Ter HaAR, D.: Condensation in Interstellar Space. Bull. Astron. Inst. Netherlands, vol. 10, no. 137, 1946, p. 371.

4. Van De Hulst, H. C.: Optics of Spherical Particles. Rech. Astron. Obs. Utrecht, vol. 11, pt. 1, 1946.

5. VAN DE Hulst, H. C.: The Solid Particles in Interstellar Space. Rech. Astron. Obs. Utrecht, vol. 11, pt. 2, 1949.

6. Hoyle, F.; and Wickramasinghe, N. C.: On Graphite Particles as Interstellar Grains. Roy. Astron. Soc., Monthly Notices, vol. 124, 1962, p. 417.

7. FranK, F. C.: Disc. for Soc. No. 5 , vol. 48,1949 , p. 67.

8. Hirth, T. P.; and Pound, G. M.: Condensation and Evaporation. Macmillan Co., New York, 1963.

9. ANON.: Formation and Trapping of Free Radicals (A. N. Bass and H. B. Broida, eds.), Academic Press, New York, 1960.

10. SchwarTzschild, M.: Structure and Evolution of Stars. Princeton University Press, Princeton, New Jersey, 1958.

11. Schwartzschild, M.: In the Distribution and Motion of Interstellar Matter in Galaxies. (L. Woltjer, ed.) W. A. Benjamin, New York, 1962, p. 266.

12. SPITZER, L.; and SAvedoff, M.: Astrophys. J., vol. 111, 1950, p. 593.

13. Frenkel, J.: Kinetic Theory of Liquids. (Ch. 7), Dover Publications, New York, 1955.

14. Tunnball, D.: In Solid State Physics, vol. 3, Academic Press, Inc., New York, 1956, p. 266.

15. Feder, J.; Russell, K. C.; Lothe, J.; and Pound, G. M.: Advances in Physics, Phil. Mag. Suppl., vol. 15, 1966, p. 111.

16. Suess, H. E.; and Urey, H. C.: Rev. Mod. Phys., vol. 28, 1956, p. 53.

17. Zemansky, M. W.: Heat and Thermodynamics. (4th ed.), McGraw-Hill Book Co., Inc., 1952.

18. States, J. C.: Introduction to Chemical Physics. MeGraw-Hill Book Co., Inc., 1939.

19. Gurney, R. W.: Introduction to Statistical Mechanics. McGraw-Hill Book Co., Inc., 1949.

20. JANAF: JANAF Thermochemical Data. Dow Chemical Co., Midland, Mich., 19611965. (Presently available from Clearinghouse for Fed. Sci. and Tech. Information, U.S. Department of Commerce, Doc. No. PB 168 370-1.)

21. Adamson, A. W.: Physicat Chemistry of Surfaces. Interscience Pub., New York, 1963. 
22. Geguzin, Y. E.; and Ovcharenko, N. N.: Soc. Phy. Uspekhi, vol. 5, 1962, p. 129.

23. Ubbelohde, A. R.; and LewIs, F. A.: Graphite and Its Crystal Compounds. Oxford Univ. Press, London, 1960.

24. Sears, G. W.: J. Chem. Phys., vol. 31, 1959, p. 358.

25. Calvert, J. G.; and PitTs, J. N.: Photochemistry. John Wiley \& Sons, Inc., New York, 1966.

26. Stecher, T. P.; and Williams, D.: Astrophys. J., vol. 146, 1966, p. 88.

27. BAtes, D. R.; and SPITzER, L.: Astrophys. J., vol. 113, 1951, p. 441.

28. Trotman-Dickenson, A. F.: Gas Kinetics. Butterworth's, Sci. Pub., London, 1955.

29. Platt, J. R.: On the Optical Properties of Interstellar Dust. Astrophys. J., vol. 123,1956, p. 486.

30. Platt, J. R.; and Donn, B. D.: Astron. J., vol. 61, 1956, p. 11.

31. Duff, Russell E.; and Bauer, S. H.: Equilibrium Composition of the C/H System at Elevated Temperatures. J. Chem. Phys., vol. 36, 1962, p. 1754.

32: Clarke, J. T.; and FoX, B. R.: Reaction of Graphite Filaments with Hydrogen above $2000^{\circ}$ K. J. Chem. Phys., vol. 46, 1967, p. 827.

33. Meyer, L.: Proceedings of Third Carbon Conference (Buffalo, New York), Pergamon Press, New York, 1959.

34. DonN, B. D.: Astron. J., vol. 70, 1965, p. 137.

35. Evans, L. F.: Requirements of an Ice Nucleus. Nature, vol. 206, 1965, p. 822.

36. Yang, L.; Simnad, M. T.; and Pound, G. M.: Acta Metallurgica, vol. 2, 1954, p. 470.

37. Donn, B. D. Astron. J., vol. 58, 1953, p. 38.

\section{DISCUSSION}

Field: With regard to your comment that interstellar material gets recycled about every $10^{6}$ years, I believe that the theory of stellar evolution, as it stands now, suggests that this recycling does occur through the red giant phase. Therefore, if nucleation takes place, it would have to take place by the agency of the red giants.

Donn: Yès, this is true. If the main ejection is from these cool stars, then one ought to look at these stellar atmospheres or clouds around these stars as being a source for the formation of possible condensation nuclei.

Field: Isn't it true that there are some observations that bear directly on the veiling of certain red giant stars?

Donn: The point that I made yesterday was that $R$ Corona Borealis shows this very precipitous drop in intensity. A suggestion which has been analyzed in detail by $O^{\prime}$ Keefe was that this may be due to the sudden condensation of carbon which forms. There has been very little further work done. G. Herbig has a paper on the spectra of $R$ Cor Bor during a decline. I looked through that and it didn't seem to me that his spectra, as I recall, at the various stages of cycle showed anything that indicates what is happening. I think that these phenomena ought to be studied observationally very carefully, as such observations are very important for understanding the cause of the decline and possible condensation processes in stellar atmospheres.

Wampler: A student in Los Angeles is working on these stars and he is trying to get the energy distribution from $4000 \AA$ to a micron. But it 
is difficult to separate reddening from temperature effects. You might have to look into some of the bands to determine temperatures.

Wickramasinghe: For the case of the gas clouds going out of red giants at $6000^{\circ}$ at the surface, one finds that you don't have to go up to $2700^{\circ}$ in order to find condensation, or in order for the vapor pressure to exceed the gas pressure. Therefore, the gas clouds undergo an adiabatic expansion.

Donn: Yes. As a matter of fact, I think that an interesting point that Dr. Hudson introduced in our discussions is that adiabatic expansion of a nozzle is one of the new techniques that shows very great applicability of studying homogeneous nucleation. In a sense the atmosphere is an adiabatically expanding cooling cloud, and this is often very favorable.

Hudson: This technique is making possible use of the adiabatic expansion in the nozzle area to study the nucleation of various gases. Both the crystal nucleation and the growth theory assume that once a molecule reaches a growth site near the nucleus or a macroscopic crystal, it is immediately incorporated. It is an additional complication that you might have a thermal activation barrier to incorporate at a growth center.

Nandy: Do you account for the change of density of the interstellar medium? A star is formed and the metal enrichment changes as a constant with time. When you consider the figure of $10^{8}$ you have to account for this change.

Donn: You mean a change in the composition due to the heavy elements formation?

Nandy: Yes.

Donn: Strictly, of course, one ought to worry about all these things, but the problem is complicated enough without that. As I recall the evidence on the building up of heavy elements, the interstellar medium in the B stars in $10^{8}$ years doesn't show any marked enhancement.

Wickramasinghe: $I$ think there is very strong evidence that the ultraviolet emission from carbon stars is very low.

Donn: I don't think the problem is solved; one needs to look at further refinements and developments of the problem. One of the disturbing things is that the advancements in our understanding of the theory of interstellar grains that have taken place in the last quarter of a century have been relatively small. The idea of grain formation in carbon stars is certainly a significant contribution.

Hemingway: One of the characteristics that we have observed in the high-altitude particles is that when we heat them in an electron beam they appear to be unstable; that is, a phase change appears to take place. After this, electron diffraction which can give a particle chemical analysis is seen. Can you shed any light on why such instability can occur? 
Donn: It is possible to form a nucleus that would grow an amorphous particle rather than a crystal; it ought to be spherical, because then there is no favored direction of growth. The formation phase is usually an unstable one. Now, if one heated the particle and annealed it, he would expect it to recrystallize. The question is: what were the conditions under which these initially were formed without a crystal structure? This is the interesting aspect.

Field: I do not understand the exponential growth. Could you explain that very simply?

Donn: It is an exponential growth as long as the particle grows by adding at an edge the material striking the surface. Larger surfaces collect more material and, therefore, growth is faster. The rate of growth is thus exponential. This procedure concerns growing a plate or a needle; it will not work for a sphere. It applies if the material that falls on the surface adds on in the same direction. It only works in a limited region because there is only a certain mean life for diffusion before the particle evaporates. Once the crystal grows larger than this, then linear growth will occur because the material that imparts at a distance that is greater than the diffusion distance will not reach the edge. One of the reasons that very large particles are unrealistic is that the mean free path of the material is much less than this distance.

Wickramasinghe: Did you try to redo the ter Haar computations on the rate of formation of triatomic molecules?

Donn: No. I assumed that the estimate ter Haar made was not altogether unreasonable and used it. (In the paper the rate of formation of triatomics is based on Bates' diatomic rate and its extension using unimolecular decomposition theory.)

Wickramasinghe: In spite of this, do you find that the nucleation rate is very small?

Donn: I think it would be too small by this process unless there are some factors that have not been accounted for.

Wickramasinghe: $I$ tried to use the Bates-Spitzer data on the recombination coefficient and found that the rate of formation of absorbed $\mathrm{CH}$ was in error by a factor of $10^{5}$.

Donn: Yes, I think it will be small. I think if the process does occur, it would probably go through an ion-molecular reaction like $\mathrm{CH}^{+}$ $+\mathrm{H} \rightarrow \mathrm{CH}_{2}^{+}$for which the cross section can be a factor from 10 to 100 larger than straight kinetic cross sections because of the polarization effects.

Lind: Is it impossible for whisker-type growth to occur in interstellar space?

Donn: A platelet is essentially a form of whisker growth. Whisker growth implies that growth is taking place along a single axis in which there is a screw dislocation; that is, a trapping center, which preserves 
itself as the crystal grows so that there is always a place to trap the atom and it keeps growing. Now, if there is a two-dimensional set of dislocations growing out, a platelet results. For three-dimensional arrays a more equiaxial crystal would be the result.

We did some scattering measurements on zinc oxide particles to see how the scattering from irregular particles compares with that from spheres. These particles are grown from vapor and they look like nuclei with four needle-like arrays sticking out. One conclusion appears to be that when these particles are oriented in a random pattern the scattering measurements are similar to those of spheres of somewhat similar characteristics, if a suitable dimension parameter that is related to the shape is used. The polarization and the angular dependence of the particles are similar to those of spheres. Therefore, for reflection nebulae, for example, the use of the Mie scattering function of the spheres is useful even though the particles may be quite irregular. Van de Hulst showed that for a cylinder, the extinction curve closely resembles that of a sphere.

I had hoped when we started these measurements that we might find some effects that deviated so markedly from spheres that something about the shapes could be determined. We were thinking here of comet tails in particular. We could discover no significant deviations, however. 